\title{
Compatible Embedding for 2D Shape Animation
}

\author{
William Baxter, Pascal Barla, and Ken-ichi Anjyo
}

\begin{abstract}
We present new algorithms for the compatible embedding of 2D shapes. Such embeddings offer a convenient way to interpolate shapes having complex, detailed features. Compared to existing techniques, our approach requires less user input, is faster, more robust, and simpler to implement, making it ideal for interactive use in practical applications. Our new approach consists of three parts. First, our boundary matching algorithm locates salient features using the perceptually-motivated principles of scalespace and uses these as automatic correspondences to guide an elastic curve matching algorithm. Second, we simplify boundaries while maintaining their parametric correspondence and the embedding of the original shapes. Finally, we extend the mapping to shapes' interiors via a new compatible triangulation algorithm. The combination of our algorithms allows us to demonstrate 2D shape interpolation with instant feedback. The proposed algorithms exhibit a combination of simplicity, speed, and accuracy that has not been achieved in previous work.
\end{abstract}

Index Terms-matching, interpolation, morphing, in-betweening, cross-parameterization, multi-scale analysis, scale-space, compatible triangulation.

\section{INTRODUCTION}

W HILE techniques for 3D have great appeal, 2D tools are still used extensively in the creation of various kinds of animation. Some tasks are simply easier in 2D: the tools are simpler, the algorithms are simpler, and the content itself is simpler and, therefore, easier and faster to work with. Examples of ongoing use include digital compositing in CG productions, cel animation and web graphics, and animated billboards as impostors in complex scenes (e.g. [1]). Nevertheless, there remain many ways in which current $2 \mathrm{D}$ techniques can be improved upon.

Recently the intuitive 2D deformation algorithm from Igarashi et al.[2] was added to Adobe's After Effects to the delight of users. However, despite the many virtues of the technique, it ultimately offers a fairly limited range of deformations. Deforming a shape to match a particular silhouette, for instance, is nearly impossible. A natural evolution would be to allow the user to modify the silhouette directly, or even supply a completely different silhouette, automatically updating the shape's interior accordingly. This can be accomplished using a 2D shape morphing algorithm.

While image morphing techniques [3], [4], [5], [6], [7], which apply a warping function to a simple rectangular domain, have been widely deployed in commercial tools, shape morphing techniques, where the domain is a complex shape, have been less widely adopted. This is despite the numerous shape morphing techniques which have been proposed in research papers (e.g. [8], [9], [10], [11], [12], [13]).

We believe the lack of adoption can be attributed to lack of a sufficiently simple, efficient, and robust solution

- W. Baxter and K. Anjyo are with OLM Digital, Inc., Tokyo, Japan. E-mail: \{baxter,anjyo\}@olm.co.jp

- P. Barla is with INRIA Bordeaux University. E-mail: pascal.barla@labri.fr to the problem of establishing the correspondence, or mapping, between two irregular shapes. Previous algorithms for matching have required the user to manually specify a large number of correspondences, or made strong assumptions about the nature of the inputs (e.g. similarity of shape, or orientation, etc.) that cause the algorithms to perform poorly when not met ([14], [9], [10]). Other algorithms are simply too slow for interactive use [15], [16], [17], [18]. Many algorithms have also dealt only with inputs which are simple polygons containing a small number of well-defined edges [14], [8], [9], [11], [12], but real-world inputs arising from practical demands are far more complex than this, often containing highly detailed and/or indistinct boundaries, like clouds, trees or long hair blowing in the wind.

The main observation motivating our approach is that exact geometric algorithms are infeasible when shapes have such complex boundaries. These details are best handled in image space. Yet, drastic global geometric changes are often desired, and since these are difficult for pure image-space methods, a hybrid approach is needed.

In this paper, we propose a solution to the correspondence problem that is suitable for complex 2D shapes, is fast, and requires less user interaction than previous techniques. The key philosophy behind our approach is to embed underlying shapes within looselyapproximating boundaries rather than try to exactly match the geometry feature-for-feature. Our contributions lie in three new algorithms that exhibit a combination of simplicity, speed, and accuracy that has not been achieved in previous work (see Section 2):

- A boundary matching algorithm (Section 3), that finds correspondences by quickly locating and optimally matching salient features on shape boundaries. It allows an artist to establish boundary correspondences with significantly less manual input than existing techniques. 
- A compatible simplification algorithm (Section 4) that efficiently simplifies boundaries while maintaining their parametric correspondence and embedding the full-resolution input shapes. It decouples the complexity of the final embedding from the complexity of the inputs.

- A compatible triangulation algorithm (Section 5) that extends the boundary correspondence to cover shapes' interiors. It is not only more efficient than all previous methods on practical inputs, but also significantly simpler than the previous best algorithms.

We believe the combination of these three algorithms is of significant importance for the widespread adoption of recent interpolation techniques [11], [19], [20].

\section{Previous work}

Much of the work in shape morphing falls into two categories: 2D morphing of images [5] and 3D morphing of meshes [21]. 2D shape morphing, lies in between these two, being both two-dimensional, like image morphing (e.g., [3], [4]), while having geometry which must be taken into account, as in mesh morphing (e.g., [22], [23], [24]). Our approach takes full advantage of the 2D nature of the problem, in a way similar to previous work such as [9], [10], [11], [12].

Shape matching Elastic distance methods have been a popular way to match shape boundaries (e.g.,[14], [10], [25]). These techniques are fast and simple, but require parameters to balance curvature error against stretching error. It is difficult or impossible to find parameters that work well for a wide variety of inputs. Moreover, they work poorly in the presence of noise (see Fig. 2(c)). In the literature on content-based image retrieval [26], a number of methods based on the medial axis have been proposed (e.g, [15], [18]); however, the medial axis is sensitive both to noise and to slight variations in shape, and methods for robust matching based on the medial axis are slow and difficult to implement.

Curvature scale-space is an effective way to deal with noise, and sophisticated algorithms have been proposed using both a full scale-space [16] and just feature points [17]. However, these methods are also too computationally demanding for interactive applications. Our approach combines the simplicity and efficiency of elastic distance with the robustness of scale-space feature-based methods.

Shape simplification Many techniques have been developed for geometric simplification of 2D lines and polygons (e.g. [27], [28], [29]). These methods find an approximation that minimizes deviation in terms of Euclidean or perceptual metrics. However, we seek not only to minimize deviation, but also to completely enclose, or embed, the full-resolution input shapes, while additionally preserving their parametric correspondence.

For 2D boundaries, [10] performs compatible simplification by subsampling, with the predictable result that embedding is lost. In [11], [22] meshes are simplified compatibly, but the former does not simplify the boundary, and the latter does not embed. The progressive hull of [30] provides an embedding simplification for 3D meshes that is similar to our method, though it only applies to a single input and is based on edge collapses rather than vertex removals. Our vertex removals lead more naturally to a compatible, parameterization-preserving simplification. We are not aware of any previous simplification algorithms which both generate an embedding and work compatibly on multiple inputs. We believe our algorithm to be the first.

Shape triangulation Previous methods for compatible triangulation generally either map polygons onto a common domain [31], [10], [11], or use divide-andconquer compatible partitioning [32], [12]. The first category is conceptually simple but not optimal in terms of runtime $\left(O\left(n^{3}\right)\right)$ or in number of Steiner vertices introduced $\left(O\left(n^{2}\right)\right)$. The latter methods are better $\left(O\left(n^{2} \log n\right)\right.$ time and often no Steiner vertices), but are algorithmically quite complex.

Our algorithm uses recursive partitioning like the latter. Efficiency is similar $\left(O\left(n^{2}\right)\right.$ on typical inputs, $O\left(n^{3}\right)$ generally), and it also generates outputs with nearminimal Steiner vertices, often zero. Yet it is significantly simpler. Though the Surazhsky-Gotsman algorithm [12] is already a simplification of a previous algorithm [32], it still requires implementation of many non-trivial data structures and algorithms described in [33], [34], [35], [36] in addition to the $O(n)$ triangulation of either [37] or [38]. In contrast, our algorithm requires no subroutine more complex than vertex visibility [39], [40], for which code can be readily be found on the web (e.g. [41]). Furthermore, based on our experience, the reliance on only very simple geometric visibility calculations also appears to have the merit of making our algorithm more robust in the face of complex inputs.

\section{BOUNDARY MATCHING}

Our approach to boundary matching is a compromise between the robustness of curvature scale-space analysis and the efficiency of elastic curve distance methods. It works in two steps. First, we locate the set of feature points along each shape boundary by extracting stable curvature extrema. This is done by analyzing extrema "signatures" through scale-space. Second, we use a new feature-based elastic curve distance, which finds automatic correspondences between feature points on each shape boundary.

Compared to previous work, the first step is faster due to our simple signature measure, while being easier to implement and similarly robust to noise. The second step is as fast as previous methods, but requires no complex parameter tuning, less user input, and is more accurate since the matching is guaranteed to go through feature points. 


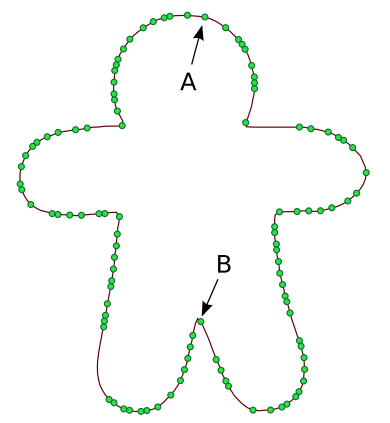

(a)

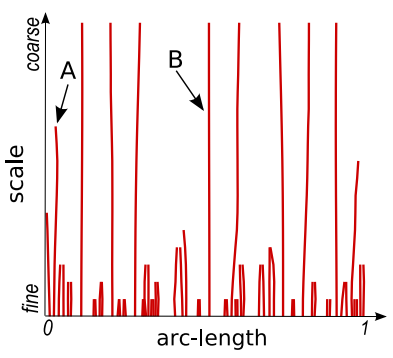

(b)

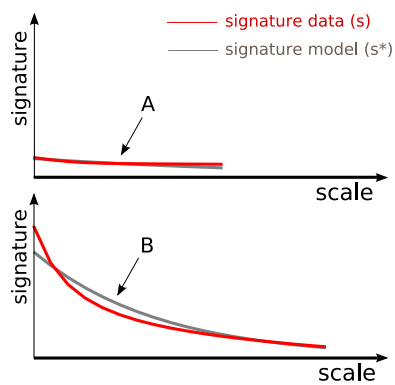

(c)

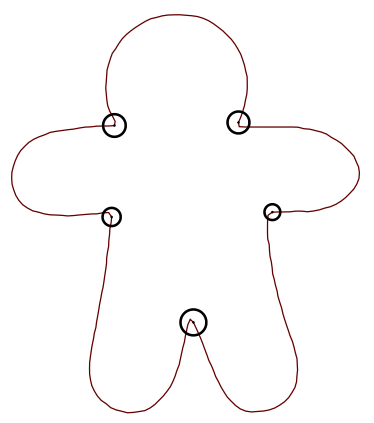

(d)

Fig. 1. Boundary analysis: (a) A boundary extracted from an image: discretization causes many fine-scale curvature extrema (green dots). (b) The fingerprint image of curvature extrema across scales. Stability of extrema A and B cannot determined from this fingerprint data alone. (c) Comparing signatures: $B$ (bottom) exhibits behavior characteristic of stable extrema; A does not. (d) Circles indicate feature points extracted by our signature analysis, with radii proportional to feature strength.

\subsection{Finding feature points}

Our first step is to find feature points on each shape to serve as candidate correspondence points (Fig. 1). These points are curvature extrema, which represent boundary concavities (minima) or convexities (maxima). However, noise and detailed variations in line style tend to create many such extrema that do not correspond to an intuitive notion of "feature" (Fig. 1(a)). Smoothing can help with the noise problem, but the difficulty is in choosing a proper scale for the smoothing.

A robust alternative consists in analyzing a boundary curve smoothed at multiple scales to find stable feature points. This is the approach taken in scale-space methods, where stable feature points are found by tracking boundary extrema at increasing scales. In the original approach of Mokhtarian et al. [16], the tracked extrema are inflection points (zero-crossings of curvature). More recently, Zabulis et al. [17] have argued that curvature extrema lead to more intuitive feature points; they show how the evolution of these extrema through scale reveals the most important structures of a shape's boundary. Fig. 1(b) shows such an evolution with a so-called "fingerprint" image, where each curve corresponds to a single extremum.

While these methods deal with noise robustly, they are also quite slow, requiring up to a minute to find features in our tests. This is because they detect stable features by computing the scale-space out to very coarse scales. Our key observation is that stable extrema have a specific behavior along the scale dimension: their curvature starts with a peak, and is progressively smoothed out (Fig. 1(c)). We call this the extremum's signature function. We assert that the signatures of stable extrema resemble decaying exponentials (Fig. 1(c),bottom). This can be understood by noting that sharp boundary features resemble delta functions in a curvature plot, and thus decay exponentially with increasing scale. In contrast, unstable extrema typically have flat or non-decaying signatures (Fig. 1(c),top).
Using our signature analysis, we can detect stable features reliably at much finer scales and thus our compute times rarely exceed a second. Moreover, the analysis also produces a measure of feature strength that is valuable for the subsequent matching step of our algorithm.

Computing signatures: We begin with a densely sampled, closed polyline with uniform arc-length parameterization. This can be extracted from an image using standard techniques. We then build a curvature scalespace and track curvature extrema at increasing scales as in [17]. As proposed in [16], we extract extrema only in the vicinity of extrema found at the previous finer scale, which significantly speeds up the process. Our contribution is in the final step where we analyze signatures to determine feature strengths and reject weak extrema, as explained below.

To decide whether an extremum is a good candidate feature point, we analyze its signature function. For feature extraction we use curvature magnitudes, disregarding sign. To ease the analysis and improve numerical stability, we map curvature magnitudes from $[0 . . \infty)$ to $[0 . .1)$ using the tanh function. The particulars of the remapping function are not critical since ultimately we only consider curvature magnitudes relative to one another (see Section 3.2). We thus take an extremum's signature data to be $s\left(\sigma_{i}\right)=\left|\tanh c\left(\sigma_{i}\right)\right|$ where $c\left(\sigma_{i}\right)$ is the curvature measured at the discrete scale $\sigma_{i}$ (red curves in Figure 1(c)).

In order to compare a curvature extremum to the ideal, we fit the measured data to an exponential signature model of the form $s^{*}(\sigma)=\exp (a x+b)$. We immediately reject any extrema with $a \geq 0$ (Fig. 1(c),top). We fit using a weighted least-squares minimization of an error function of the form $e(a, b)=\sum_{i=1}^{N} w\left(\sigma_{i}\right)\left(a x+b-\log s\left(\sigma_{i}\right)\right)^{2}$, with $w(x)=((N-x) / N)^{2}$, so that values near the minimum scale (where the shape is less smoothed out) have more influence. As mentioned, we use $a$ to reject unstable extrema, but we also use $b$, to determine the feature strength. The feature strength $f$ is defined by its 


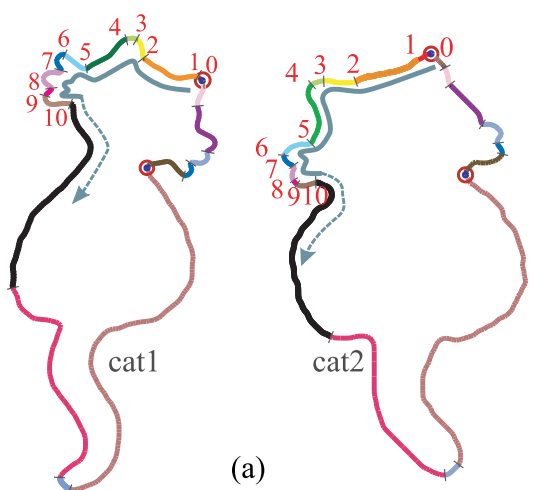

(a)

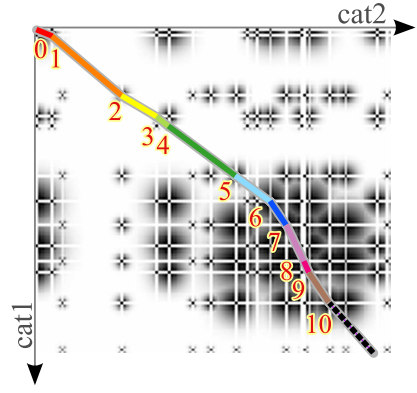

(b)

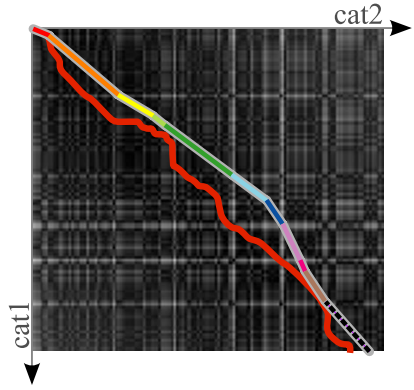

(c)

Fig. 2. Boundary matching: (a) Two input shapes and their boundary correspondence computed with our matching algorithm. User correspondences are circled in red, while automatic correspondences are identified by numbers, and delimit boundary segments of similar color. (b) Cost matrix of our approach for a subset of the boundary: dark blobs represent attractors around potential correspondences and the matching path goes exactly through curvature extrema. (c) Cost matrix for curvature-based matching: the path (in red) does not go exactly through the desired correspondences and is sensitive to noise.

peak value, the value at $x=0$, i.e. $f=s^{*}(0)=\exp (b)$. Figure 1(d) shows the feature points extracted with our approach. The radius of each circle locator is proportional to the strength of the corresponding extremum.

\subsection{Extracting automatic correspondences}

From the multi-scale analysis, for each shape we get a set of feature points along with their strength and sign of curvature (indicating convexity). We normalize feature strengths by dividing by the maximum strength value over the shape pair, and we use a small threshold $\left(f_{\min }=\right.$ 0.05 ) to discard extrema that obviously come from noise, but we keep most of the extrema. The main purpose of our elastic matching algorithm is then to make use of this information to automatically extract meaningful correspondence points. The full boundary mapping is obtained by establishing a uniform parameterization inbetween each successive pair of correspondences (Fig. 2). The only information a user need specify is the first pair of correspondence points.

The problem of finding a boundary mapping is then solved using a dynamic programming approach: a cost matrix is built where each cell stores the dissimilarity between every possible pair of points on each boundary, and the dynamic programming algorithm finds a matching path of least cost (Fig. 2(b-c)) that links the first pair of points to the last pair (i.e., user-given correspondences). Classic curve matching techniques (e.g.[14], [10], [25]) set up a cost matrix where each individual cost is based on an arc-length, curvature or orientation dissimilarity (Fig. 2-c). Instead of using raw curvature, orientation or arc-length as features to be matched, we use the feature strength derived in Section 3.1.

We require that both features be strong in order to consider the pair a potential correspondence. We turn this constraint into a confidence measure defined by $c(p, q)=\min \{f(p), f(q)\}$ where $p$ and $q$ are two points on different boundaries, $f$ is the feature strength. The min function acts as a fuzzy AND operator. Moreover, we enforce $c(p, q)=0$ whenever $p$ and $q$ are not feature points or have opposite signs of curvature. Our goal is then to make use of this confidence measure to build a cost matrix, with the additional constraint that the matching runs exactly through automatically selected pairs of feature points, and that each feature point is used at most once.

Building the cost matrix: The intent is that the higher the confidence value, the more likely the matching will go through it. This is translated to a cost measure by first filling the cost matrix with a maximum cost of 1 for each possible pair of points, and then positioning inverted Gaussian blobs everywhere $c(p, q) \neq 0$. These blobs act like "attractors" as they reduce costs toward 0 around the center of the Gaussian (Fig. 2(b)). The standard deviation of a Gaussian blob corresponds to the attraction strength of a candidate correspondence, so we make it proportional to the confidence measure: $\sigma(p, q)=\frac{1}{K} c(p, q)$. The constant $K$ is used to normalize attractors' influence, and we make it equal to the minimum of average confidences in each shape $K=\min \left\{\frac{1}{n} \sum_{p} f(p), \frac{1}{m} \sum_{q} f(q)\right\}$. This heuristic proved to work well in all our examples. Moreover, to ensure that the matching runs exactly through selected correspondences, we penalize point pairs where only one point is a feature, setting the cost to 1 . This corresponds to the white horizontal and vertical lines in Figure 2(b).

Comparison of matching paths in Figure 2(b-c) demonstrate the advantage of our boundary matching procedure: it extracts significant correspondences more accurately, while requiring no parameter tuning and being fast enough for interactive editing. Perhaps more importantly, in tests we were always able to find a satisfactory matching with our algorithm, which was not the case with the other algorithms we evaluated. More 


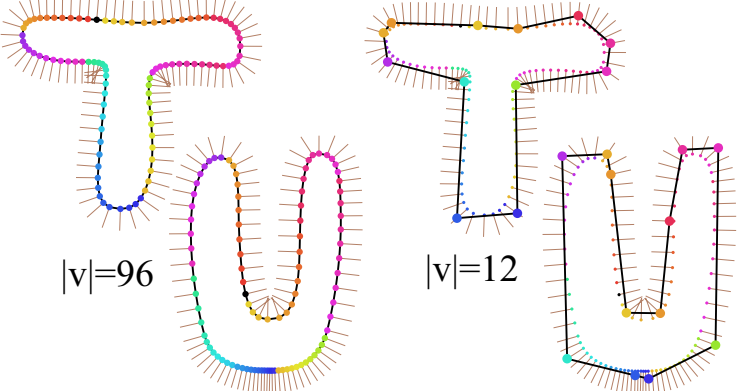

Fig. 3. Compatible simplification: A result from our compatible, embedding, parameterization-preserving boundary simplification. (left) Initial shapes. (right) Simplified shapes.

results and comparisons can be found in Section 6 .

\section{COMPATIBLE BOUNDARY SIMPLIFICATION}

The output of the matching algorithm of the previous section is a densely sampled pair of corresponding boundary polygons. For generating the subsequent compatible tessellation it is preferable to work with a simplified version of this matching, but the simplification must maintain parametric correspondence while still completely enclosing the original shapes. To satisfy these requirements, we present a new algorithm for compatible boundary simplification (Fig. 3).

The key idea is that when vertices are removed through simplification, we restore the local embedding by moving neighboring vertices along their normals. In particular, for maximal efficiency we consider moving only the immediate neighbors of a removed vertex. Moving normal to the boundary is the key element that serves to preserve the parametric identity of each point.

We follow the general structure of progressive mesh simplification [42], assigning costs to each simplification operation-in our case vertex removals-and using a priority heap with greedy selection to generate a simplification sequence. The cost of each vertex removal operation is taken to be the change (increase) in polygon area that will result (see Fig. 4). For the overall compatible simplification cost we take the maximum cost of the two (inputs are normalized to have approximately the same scale). Once the removal sequence is computed, the heap may be discarded, and the recorded collapse sequence can be used to quickly switch between different simplification levels. In practice, the time spent for simplification is negligible in the overall pipeline, but it significantly speeds up the subsequent triangulation.

Restoring local embedding: After removing a vertex, $p_{0}$, we need to restore the embedding property for convex vertices. Concave vertices as in Fig-

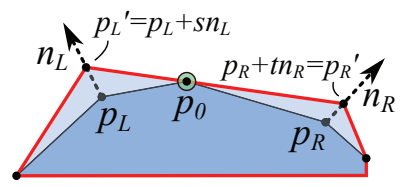
ure 4(a) can simply be removed. Figures $4(\mathrm{~b}-\mathrm{e})$ show several convex examples. (a)

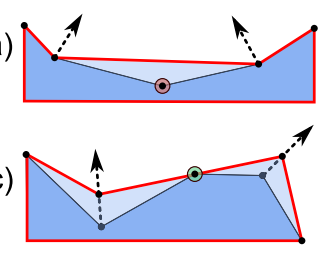

(b)

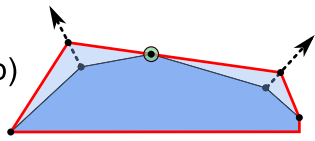

(e) (d)

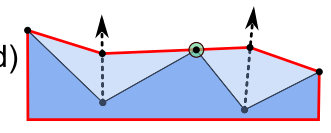

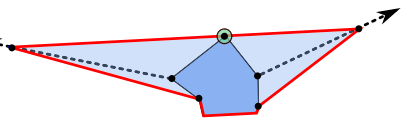

Fig. 4. Simplification via vertex removal: The original polygon (dark blue) and boundary after removal (red). The cost of a removal is taken to be the area added by the operation. Highly convex vertices like (e) are poor candidates for simplification, and the area cost metric reflects this.

As mentioned, we restore embedding by perturbing the immediate neighbors, $p_{L}$ and $p_{R}$, along their unit normals, $n_{L}$ and $n_{R}$, so that new vertex positions are $p_{L}^{\prime}=p_{L}+s n_{L}$ and $p_{R}^{\prime}=p_{R}+t n_{R}$. This gives us a two-parameter search space, $(s, t)$ for "good" vertex positions. We choose to place the vertices so as to equally distribute the amount of perturbation between $p_{L}$ and $p_{R}$ (i.e. make $s=t$ ) subject to the constraint that the segment $\overrightarrow{p_{L}^{\prime} p_{R}^{\prime}}$ intersects $p_{0}$. This leads to a quadratic equation in $s:$

$$
\begin{aligned}
0 & =s^{2}\left(n_{R} \cdot n_{L}^{\perp}\right)+\left(p_{L}-p_{R}\right) \cdot\left(p_{0}-p_{L}\right)^{\perp} \\
& -s\left[\left(n_{R}-n_{L}\right) \cdot\left(p_{0}-p_{L}\right)^{\perp}+\left(p_{L}-p_{R}\right) \cdot n_{L}^{\perp}\right],
\end{aligned}
$$

where $(x, y)^{\perp}=(y,-x)$. The minimum positive root is the solution sought. If no positive root exists it means the normals are separated by an angle of greater than 180 degrees. In these cases we relax the normal direction constraint. We do this by turning both normals inward by a fixed amount (we use a step size of 45 degrees) and trying the procedure again. Each time we turn the angles inward, we add a large penalty to this vertex's removal cost to ensure we use collapses that do not require going off-normal first.

Modifications: First, we can obtain an inscribed simplification (right) simply by reversing the direction of the boundary normals used. Second, the algorithm trivially generalizes to $N$-way compatible simplification by taking the maximum of $N$ vertex costs rather than of two.

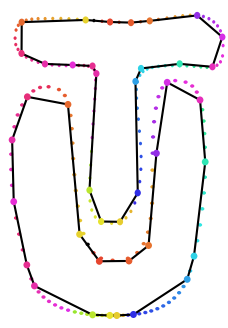

\section{COMPATIBLE TRIANGULATION}

We establish interior correspondence using compatible triangulation. Given two polygons with compatible boundaries as input, we recursively and compatibly partition the two polygons using compatible link paths as in [32], [12]. Recursion on a branch terminates when both inputs are triangles, which have a trivial linear mapping. We introduce a new algorithm for finding 


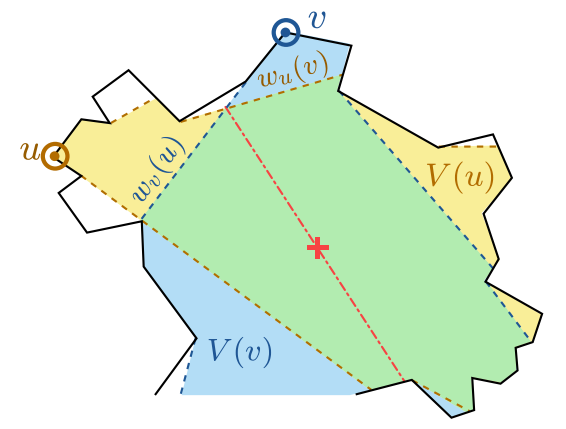

Fig. 5. Visibility polygons and windows: The visibility polygons $V(u)$ and $V(v)$ for vertices $u$ and $v$ and the windows $w_{u}(v)$ and $w_{v}(u)$ are indicated. The red ' + indicates where our algorithm places the Steiner vertex for this case.

compatible link paths, which is significantly simpler than the previous algorithms and also more efficient on typical inputs.

Terminology: A link path is an interior polyline that joins two vertices, and compatible link paths are two such paths which join corresponding pairs of vertices in two polygons. The link distance is the number of line segments in a link polyline. As a shorthand, we refer to a link path with a link distance of $k$ as a " $k$-link". A $k$-link requires the introduction of $k-1$ Steiner vertices. The goal is to triangulate by finding compatible partitions with minimal $k$. It is beneficial to minimize the number of Steiner vertices because it reduces not only the run time and memory usage of the algorithm itself but also the run time of any subsequent mesh processing, such as non-linear optimization of parametric stretch (e.g. [43], [22]), which can be a significant bottleneck in the overall processing pipeline.

Our algorithm works in three stages. First, we compute per-vertex visibility polygons and use these to find compatible 1-links. Second, if no compatible 1link is found, we look for compatible 2-links. These can be found by looking for intersections of the vertex visibility polygons. Third, in the rare case that neither a compatible 1- or 2-link exists, we resort to a general $k$-link finding mechanism which builds pseudo-optimal $k$-links out of combinations of the existing 1- and 2links by solving the classic all-pairs-shortest-paths graph problem.

Stage 1: As a first step, we compute the visibility polygon of each vertex in each polygon [39], [40]. A visibility polygon is the set of points, all of which have direct line-of-site from some given feature (see Figure 5). A vertex visibility polygon can be computed in $O(n)$ for each of $n$ vertices, so this step requires $O\left(n^{2}\right)$ work. We also modify the basic algorithm to simultaneously record the complete $n \times n$ visibility matrix for each polygon as it proceeds, making later determination of direct visibility between any two vertices an $O(1)$ operation. In fact we record not just visibility in these matrices, but bestestimate minimal link distances between pairs of vertices
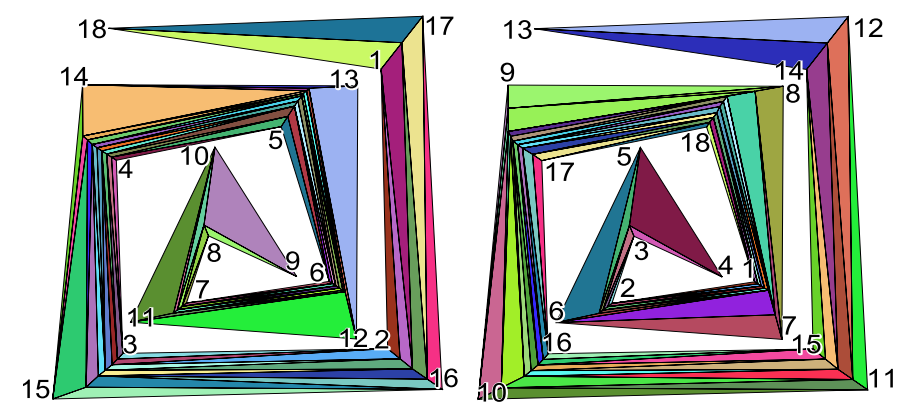

Fig. 6. Compatible triangulation torture test: Results of our algorithm on shapes that are difficult to triangulate compatibly due to necessity of $k$-links, $k>2$. Numbers indicate vertex correspondences; colors indicate triangle correspondences. 32 Steiner vertices were added.

as we find them, for use in stage 3 .

Specifically, let the two link distance matrices be $D^{0}$ and $D^{1}$. At this stage, we record a 1 in any element $D_{i j}^{0}$ or $D_{i j}^{1}$ for which vertices $i$ and $j$ have direct visibility in the corresponding shape, and $\min (|i-j|, n-|i-j|)$ for those which do not. The latter is simply the distance from $i$ to $j$ walking along the boundary in the shorter direction. We refer to these as trivial $k$-links. Partitioning using these is not generally useful, so is to be avoided. But they serve as an upper bound on the link distance between any two vertices.

The approach is greedy. If we can find a corresponding pair of vertices in each polygon that have direct visibility (i.e. the pair is a 1-link in both polygons), then we use it. Given the visibility matrix from the preprocess, all pairs can be easily checked in $O\left(n^{2}\right)$ time. Often there are many compatible 1-links. In this case, like [12], we choose a pair that results in the most balanced split of the polygons in terms of number of vertices on each side.

For practical inputs, a compatible 1-link can almost always be found; however, Steiner vertices must sometimes be added to one or both polygons to create a compatible partitioning (see Figure 8, or Figure 6 for a particularly challenging case). If no compatible 1-links are found then we proceed to stage 2 .

Stage 2: The second stage of our algorithm looks for vertex pairs $(u, v)$ with minimal link distance of two, or 2-links. 2-link visibility means that there is some point $q$ in polygon $P$ which is visible from both vertex $u$ and vertex $v$. The locus of such points is the intersection of the visibility polygons for $u$ and $v$. Thus all 2-links could be found by calculating the intersections of the vertex visibility polygons. However, polygon intersection in general requires $O\left(n^{2}\right)$ time, so this would lead to $O\left(n^{4}\right)$ complexity to test all pairs. Fortunately, for this case we can test for intersection in $O(1)$, by simply intersecting two particular edges.

These two particular edges are the so-called windows that separate $u$ from $v$, inside $P$ (Fig. 5). A window $w_{u}(v)$ is an edge of the visibility polygon $V(u)$ that separates vertex $u$ from vertex $v$. This edge exists whenever $u$ and 
$v$ do not have direct line-of-sight visibility in $P$, and is always unique [34], [33]. The same holds for $w_{v}(u)$. Using these facts and some other results from Suri, we prove the following lemma in [44]:

Lemma 1: The two windows $w_{u}(v)$ and $w_{v}(u)$ intersect iff there exists a minimal 2-link path between $u$ and $v$.

Lemma 1 is the key insight that gives us an $O(1)$ algorithm for determining whether two visibility polygons intersect: they intersect if and only if $w_{u}(v)$ and $w_{v}(u)$ do. This leads to an overall $O\left(n^{2}\right)$ run time for stage 2 . Note that the windows separating each pair of vertices are easily found as a byproduct of the vertex visibility algorithm run in stage 1 . If a compatible 2-link is found, we place the Steiner vertex along the ray that bisects the angle between the two window edges (Fig. 5).

While performing this search we also update the visibility matrices from the first stage with the newly acquired 2-link visibility information. If no compatible 2-links are found, then we proceed to stage 3.

Stage 3: The main idea is to find compatible $k$-links where $k>2$ by simply combining the existing 1 - and 2-link paths. If we can get from $u$ to $w$ via a 2-link and $w$ to $v$ using a 1-link then we know we can get from $u$ to $v$ in 3 steps. This is the description of a classic graph distance problem. Our link distance matrix encodes the initial distances in the graph and we wish to solve the allpairs-shortest-paths problem on that graph. The FloydWarshall algorithm finds these in $O\left(n^{3}\right)$.

Once a non-trivial pair of compatible $k$-link paths is found, we place the Steiner vertices. The path consists of a combination of 1-link and 2-link portions. We first place the central vertex of the 2-link portions using the technique mentioned above. All remaining vertices lie on the polygon boundary initially and these can be adjusted towards the interior of the polygon by various means.

Extensions: The triangulations generated by our algorithm are nearly minimal; however, they usually include many long thin triangles which are undesirable for applications. To improve the mesh quality, we use a a variation of the compatible refinement scheme in [12]. Quality meshes can be generated much more efficiently working coarse-to-fine in this way than in the approaches which start with a fine mesh of low quality [31], [10], [11]. Figure 8 shows some compatible triangulations generated by our algorithm both before and after refinement. Additionally we have implemented a simple scheme for incorporating interior correspondences, similar to that of [10]. Essentially, when there are interior correspondences we perform a pre-process that eliminates the holes and interior points by cutting bridges to them.

\section{Results ANd discussion}

To demonstrate the benefits of the newly introduced algorithms, we have created a system which implements both the rigid morphing of [11] with improvements detailed in [45], as well as the rigid deformation technique

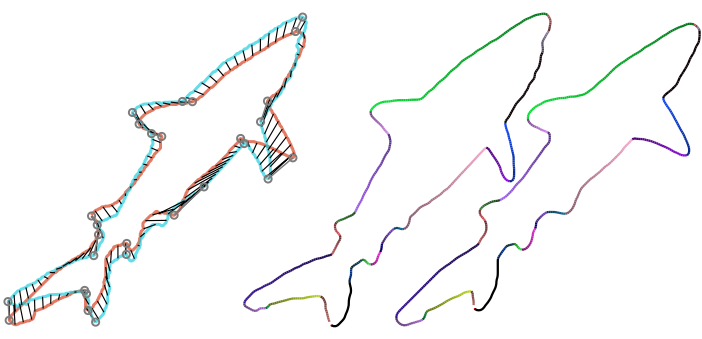

Fig. 7. Matching comparison: A comparison with a matching result taken from Zabulis et al. [17] (left) vs. our algorithm (right). No manual correspondences were specified. Our algorithm obtains very similar results but in a fraction of the time.

TABLE 1

Boundary matching comparisons

\begin{tabular}{lccc}
\hline Input & Our method & Curvature & Tangent \\
\hline Cat standing & $=2$ & $>6$ & $>6$ \\
Ant-spider & $=5$ & $>13$ & $>13$ \\
Pony & $=2$ & $=5$ & $=8$ \\
Bird & $=2$ & $=5$ & $=2$ \\
Barking dog & $=4$ & $>7$ & $>6$ \\
Elephant-giraffe & $=4$ & $>7$ & $>7$ \\
Kicking man & $=1$ & $>3$ & $>10$ \\
\hline
\end{tabular}

The number of manual correspondences required to obtain a satisfactory boundary matching. The indication " $=n$ " means it took exactly $n$ correspondences; " $>n$ " means that after $n$ manual correspondences, the result still contained regions with significant artifacts that could not be eliminated without manually specifying the correspondence of nearly every point in the region. The inputs used for the comparison are those shown in Fig. 8, from top to bottom.

of [2]. Deformation techniques can be used to create new inputs for morphing which are trivially compatible, allowing for the simple creation of animations that are impossible with either technique alone. Figures 7-12 show various results of our boundary matching, simplification, and triangulation algorithms. Videos demonstrating our system can be found at http://www.olm.co.jp/en/rd/ 2008/06/compatible_embedding.html.

Boundary matching: Though it is impossible for any automatic algorithm to guess the user's intention correctly $100 \%$ of the time, with our technique, a single user correspondence is often sufficient. Fortunately, applying the dynamic programming algorithm is also very fast (milliseconds), so users can obtain instant feedback while adding and removing correspondences. The scalespace matching algorithm of [17] can achieve similar results (see Fig. 7), but the method is not fast enough to be used interactively. On the other hand we find the previous techniques that are interactive require many more manual correspondences than our method, and often fail to produce a satisfying matching, even after manual specification of many correspondences. Table 1 summarizes these findings. It compares the number of user-given correspondences necessary to obtain a satisfying matching for three different methods: ours (featurebased), curvature-based, and tangent-based elastic curve matching. 

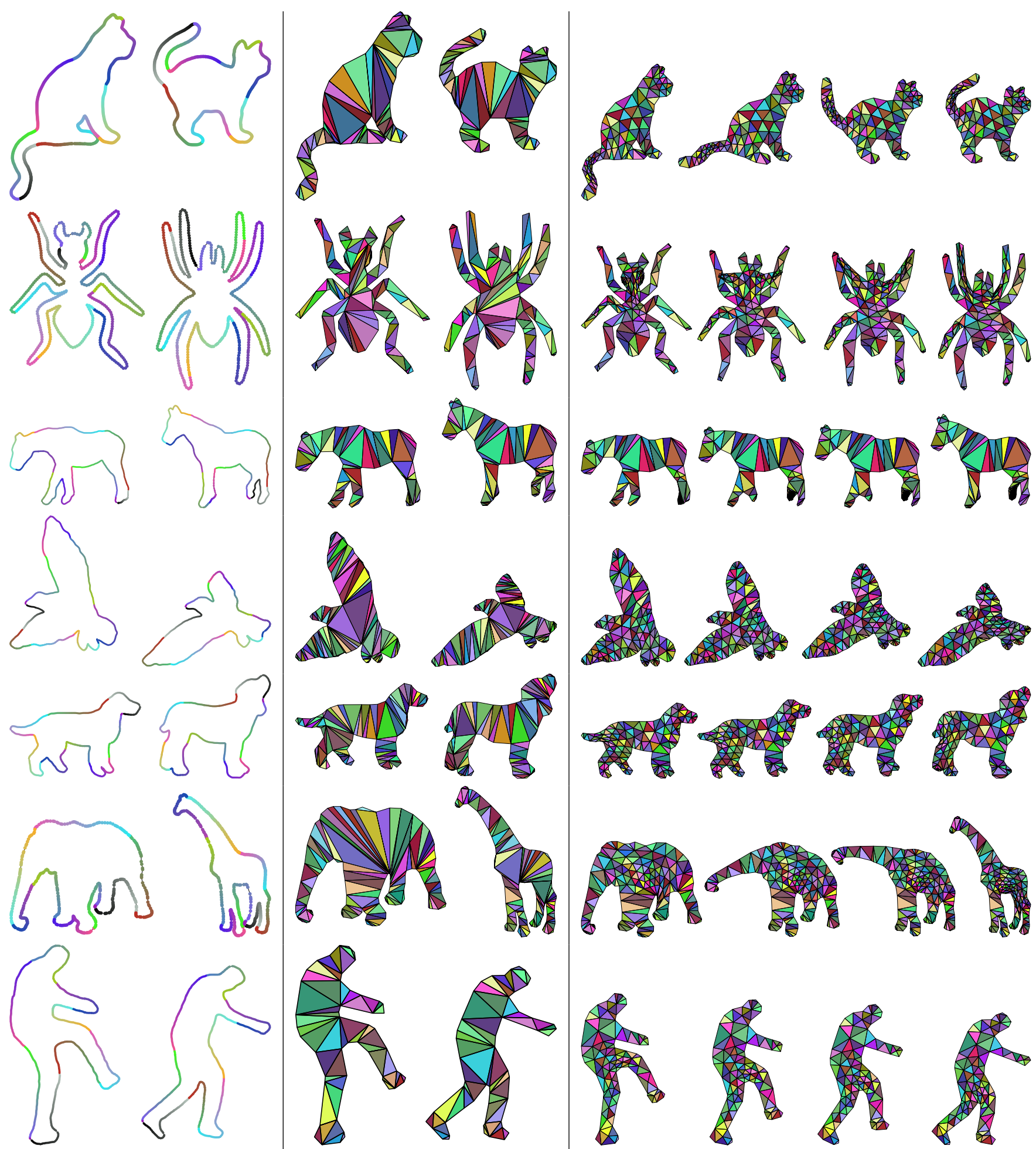

(a)
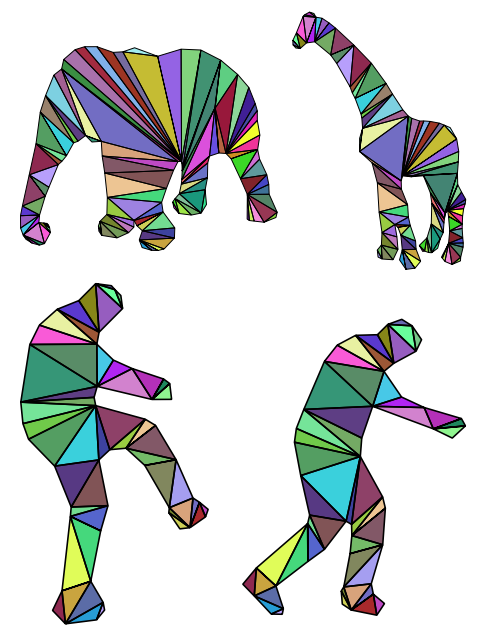

(b)
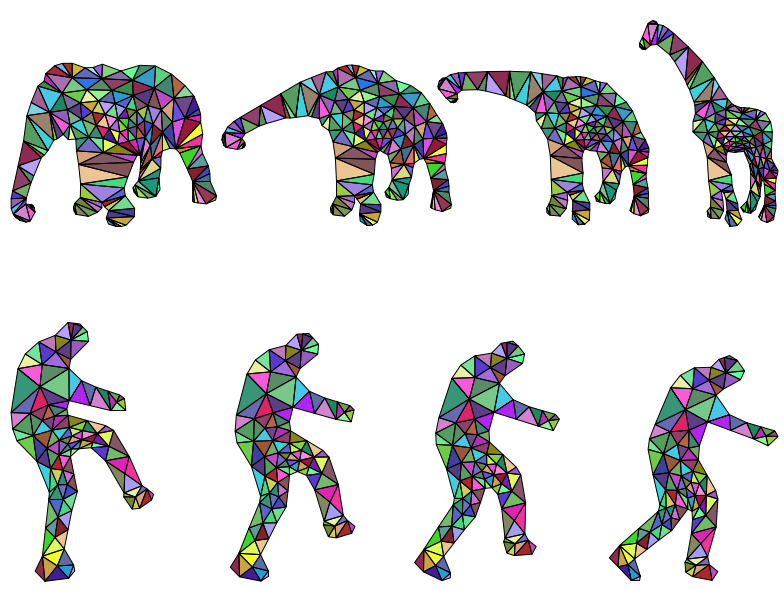

(c)

Fig. 8. Embedding results: (a) boundary matching (b) compatible triangulation of compatibly simplified boundaries (c) mesh refinement and morphing. Note how our compatible triangulation handles extreme stretching (e.g. spider shape, second row). From top to bottom, these examples required 2, 5, 2, 2, 4, 4, and 1 manual boundary correspondences, and no interior correspondences. The initial tesselations required 0,3,4,4,4,2, and 0 Steiner vertices. 


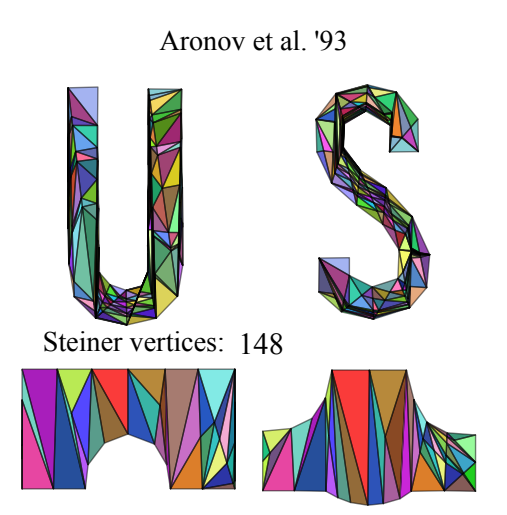

Steiner vertices:

13

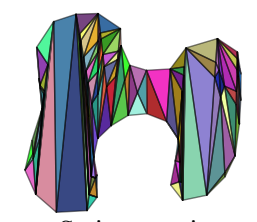

Steiner vertices:

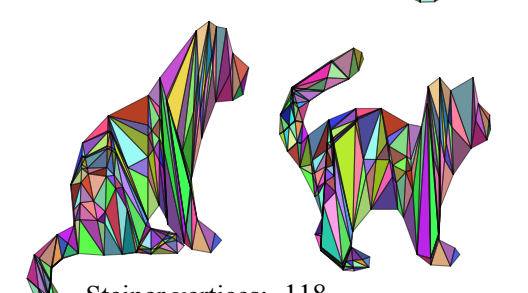

Steiner vertices: 118

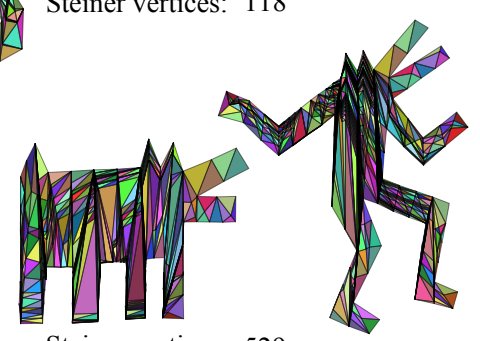

Steiner vertices: 529

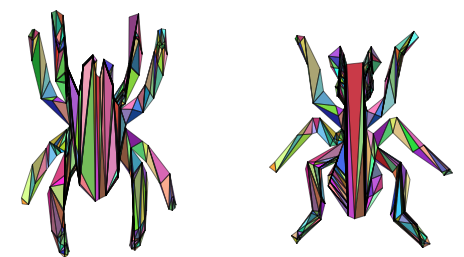

Steiner vertices: 299

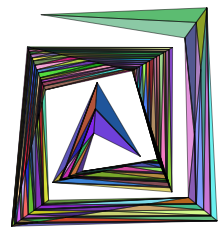

Steiner vertices: 126
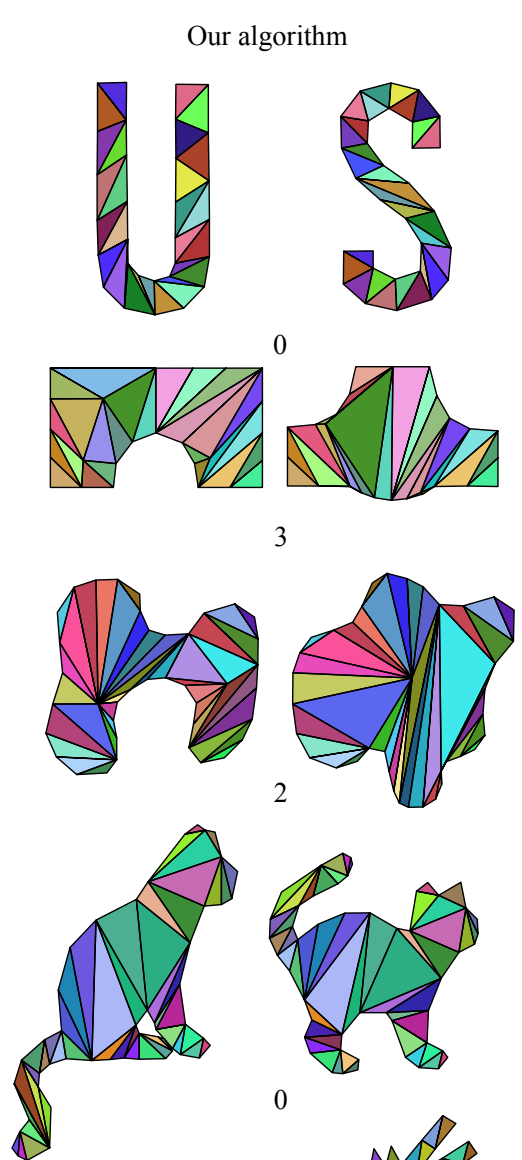

0

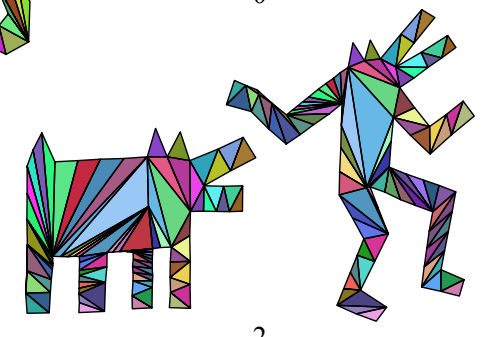

2
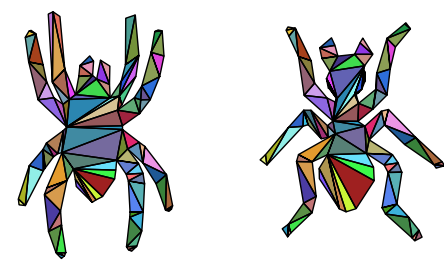

3
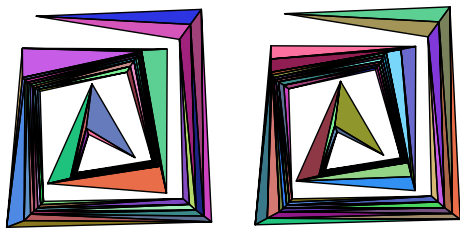

32
Surazhsky-Gotsman '04
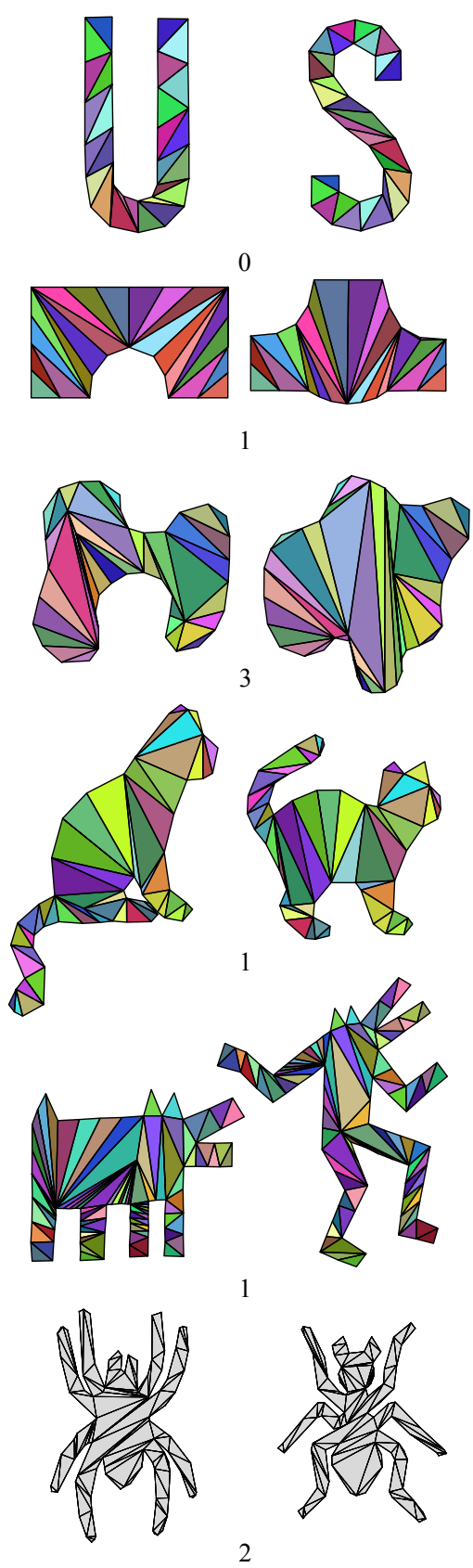

N/A

Fig. 9. Compatible tesselation comparisons: Comparing our results with those of Aronov et al. [31] and SurazhskyGotsman [12]. Our algorithm generates tesselations similar to [12], but with a significantly simpler algorithm. The last image in column 3 was created using Surazhsky and Gotsman's implementation of [12]. "N/A" indicates that both our implementation of [12] and theirs failed due to numerical robustness issues. 
TABLE 2

Tesselation and Smoothing Timing Comparison

\begin{tabular}{ccccc}
\hline Verts & Steiner verts & Smoothed verts & $\begin{array}{c}t \text { tess } \\
(\mathrm{msec})\end{array}$ & $\begin{array}{c}t \text { smooth } \\
(\mathrm{msec})\end{array}$ \\
\hline 70 & $0 / 232$ & $110 / 342$ & $14 / 25$ & $279 / 2212$ \\
81 & $0 / 294$ & $121 / 415$ & $19 / 34$ & $231 / 3406$ \\
92 & $4 / 412$ & $136 / 544$ & $24 / 48$ & $473 / 5508$ \\
110 & $0 / 580$ & $150 / 730$ & $29 / 75$ & $408 / 10723$ \\
121 & $2 / 704$ & $163 / 865$ & $41 / 98$ & $448 / 13689$ \\
140 & $3 / 975$ & $183 / 1155$ & $54 / 147$ & $521 / 17066$ \\
162 & $1 / 1394$ & $203 / 1596$ & $60 / 240$ & $627 / 34036$ \\
199 & $4 / 2043$ & $243 / 2282$ & $92 / 384$ & $686 / 46587$ \\
\hline
\end{tabular}

Timing information for tesselation and subsequent smoothing using two different tesselation techniques. We compare our algorithm with that of Aronov et al. [31]. Results are in the form (ours/Aronov). For both tesselations, smoothing was done using a variation on the method of Surazhsky et al. [12]. The test was performed on the pair of cats at the top of Fig. 8, simplified to different numbers of initial vertices by the compatible simplification algorithm of Section 4 .
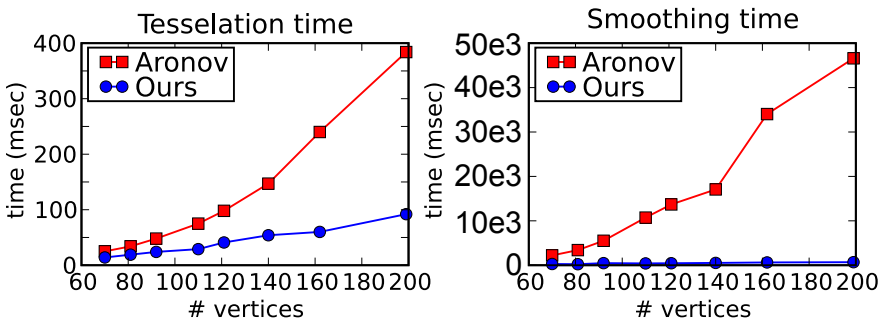

Fig. 10. Tesselation timing comparison: Plots of the left two columns of Table 2.

The first observation is that our method requires fewer user interactions than the other two (between 2 and 3 times fewer manual correspondences). However, even more important is that with our method, it was always possible to find a satisfying matching using a small number of manual correspondences. The reason is that we only do direct matching on significant features or user specified correspondences. Our technique effectively falls-back to uniform parametrizations where there are no significant features. In contrast, with matching algorithms based directly on matching curvatures, arclengths, or tangents globally, it is easy for the matching to get hung up on noisy features, or to discount good matches simply because of slight differences in feature magnitude.

Compatible triangulation: In [11] it is noted that "the most time-consuming step is optimizing triangle shape". With the combination of our boundary simplification and subsequent near-optimal triangulation, even this step (i.e. our mesh refinement based on [12]) takes just a few tenths of a second (see Table 2 and Fig. 10). In comparison, with a method that generates a large number of Steiner vertices like that of [31] used by [11], the time for smoothing easily reaches tens of seconds, which is unacceptable for an interactive application. With a more complex smoothing metric to reduce the cross-distortion, as in [30], the difference would likely be even more dramatic.

Regarding simplicity of implementation, it took one co-author of this paper two days to implement [31], one week for our new algorithm, and three months to implement [12]. In fact, we have subsequently learned that even the authors of [12] themselves did not implement the Suri minimal-link algorithm described in the paper, because it was deemed too complex [46]. Though [31] is clearly the simplest of the algorithms, the time required to smooth the resulting meshes can be prohibitive. On the other hand, the relatively complicated data structures involved in [12] proved challenging to implement and debug, and appear to be more prone to numerical degeneracies than our algorithm, as can be seen in Fig. 9.

\section{CONCLUSIONS AND FUTURE WORK}

We presented a set of algorithms for the compatible embedding of 2D shapes, a necessary intermediate step for the morphing of practical inputs having complex silhouettes. Fig. 12 demonstrates the effectiveness of our coarse embedding approach on several such shapes with relatively complex silhouettes.

The major advantage of our approach is that it allows a user to create shape morphings easily and efficiently with just a few clicks. Each step in our processing pipeline is fast enough to provide a user with a truly interactive system for viewing and modifying results, which is important for the intended use as part of an animation system. A second significant advantage is the simplicity of each of the algorithms.

However, our current system has some limitations. Our full vision of a compatible embedding animation system has yet to be realized. In this paper we focused on the geometric aspects of the problem, neglecting imagespace. Thus, for example, some "ghosting" artifacts can still be seen in our results that use texture cross-fading. Our geometric techniques handle the large-scale mapping between shapes, but ultimately the finer details, the "residual", should be handled in image space, perhaps by warping the underlying textures, or by more sophisticated texture blending schemes. We are also interested in improving our approach to better handle shapes of varying genus, though the compatible embedding already gives us a way to handle small differences by simply treating holes as texture.

Additionally, our current approach only works for a pair of inputs. For more complex animations it would be advantageous be able to construct compatible embeddings of several shapes at a time and morph between them as in [24], [47]. We are currently in the process of implementing this extension.

From a user-interface standpoint, it would be beneficial to have an advanced layering system that simplifies assembly of multiple compatible embeddings into composite animations. Also useful would be a boundarybased deformation tool similar to [13]. Such features would greatly expand the creative potential for users, leading to a qualitatively new approach to the creation of $2 \mathrm{D}$ animations. 
Also, we have assumed a texture-based representation of the details. However, the parameterization provided by the underlying compatible triangulation can also be used as a coordinate system for vector drawing elements. Thus compatible embeddings can also be used for interpolation-based animation of vector drawings.

\section{ACKNOWLEDGMENTS}

This work was supported in part by the Japan Science and Technology Agency, CREST project.

\section{REFERENCES}

[1] L. Kavan, S. Dobbyn, S. Collins, J. Zara, and C. O'Sullivan, "Polypostors: 2D polygonal impostors for 3D crowds," in 2008 ACM SIGGRAPH Symposium on Interactive 3D Graphics and Games, February 2008.

[2] T. Igarashi, T. Moscovich, and J. F. Hughes, "As-rigid-as-possible shape manipulation," Proc. SIGGRAPH '05, pp. 1134-1141, 2005.

[3] T. Beier and S. Neely, "Feature-based image metamorphosis," in Proc. SIGGRAPH '92, New York, NY, USA, 1992, pp. 35-42.

[4] S.-Y. Lee, K.-Y. Chwa, and S. Y. Shin, "Image metamorphosis using snakes and free-form deformations," in Proc. SIGGRAPH '95, 1995, pp. $439-448$.

[5] G. Wolberg, "Image morphing: A survey," The Visual Computer, vol. 14 , no. 8 , pp. $360-372,1998$

[6] S. Schaefer, T. McPhail, and J. Warren, "Image deformation using moving least squares," in Proc. SIGGRAPH '06, 2006, pp. 533-540.

[7] H. Fang and J. C. Hart, "Detail preserving shape deformation in image editing," Proc. SIGGRAPH '07, vol. 26, no. 3, p. 12, 2007.

[8] T. W. Sederberg, P. Gao, G. Wang, and H. Mu, "2-D shape blending: an intrinsic solution to the vertex path problem," in Proc. SIGGRAPH '93, 1993, pp. 15-18.

[9] E. Goldstein and C. Gotsman, "Polygon morphing using a multiresolution representation," in Proc. Graphics Interface, 1995, pp. 247-254.

[10] A. Tal and G. Elber, "Image morphing with feature preserving texture," in Computer Graphics Forum (Eurographics '99), vol. 18(3), 1999, pp. 339-348.

[11] M. Alexa, D. Cohen-Or, and D. Levin, "As-rigid-as-possible shape interpolation," in Proc. SIGGRAPH '00, 2000, pp. 157-164.

[12] V. Surazhsky and C. Gotsman, "High quality compatible triangulations," Eng. w. Computers, vol. 20, no. 2, pp. 147-156, Apr. 2004.

[13] M. Eitz, O. Sorkine, and M. Alexa, "Sketch based image deformation," in Proc. Vision, Modeling and Visualization, 2007, pp. 135-142.

[14] T. W. Sederberg and E. Greenwood, "A physically based approach to 2-D shape blending," in Proc. SIGGRAPH '92, 1992, pp. 25-34.

[15] T. B. Sebastian, P. N. Klein, and B. B. Kimia, "Recognition of shapes by editing their shock graphs," IEEE Trans. Pattern Anal. Mach. Intell., vol. 26, no. 5, pp. 550-571, 2004.

[16] F. Mokhtarian and A. K. Mackworth, "A theory of multiscale, curvature-based shape representation for planar curves," IEEE Trans. Pattern Anal. Mach. Intell., vol. 14, no. 8, pp. 789-805, 1992.

[17] X. Zabulis, J. Sporring, and S. C. Orphanoudakis, "Perceptually relevant and piecewise linear matching of silhouettes," Pattern Recognition, vol. 38, no. 1, pp. 75-93, 2005.

[18] M. van Eede, D. Macrini, A. Telea, C. Sminchisescu, and S. S. Dickinson, "Canonical skeletons for shape matching," in ICPR '06: Proceedings of the 18th International Conference on Pattern Recognition, Washington, DC, USA, 2006, pp. 64-69.

[19] C. Gotsman and V. Surazhsky, "Guaranteed intersection-free polygon morphing," Computers and Graphics, vol. 25, no. 1, pp. 67-75, 2001.

[20] T. Surazhsky, V. Surazhsky, G. Barequet, and A. Tal, "Blending polygonal shapes with different topologies," Computers and Graphics, vol. 25, no. 1, pp. 29-39, 2001

[21] M. Alexa, "Recent advances in mesh morphing," Computer Graphics Forum, vol. 21, no. 2, pp. 173-197, 2002.

[22] J. Schreiner, A. Asirvatham, E. Praun, and H. Hoppe, "Intersurface mapping," Proc. SIGGRAPH '04, pp. 870-877, 2004.
[23] D. Xu, H. Zhang, Q. Wang, and H. Bao, "Poisson shape interpolation," in SPM '05: Proceedings of the 2005 ACM symposium on Solid and physical modeling, 2005, pp. 267-274.

[24] R. W. Sumner, M. Zwicker, C. Gotsman, and J. Popović, "Meshbased inverse kinematics," in Proc. SIGGRAPH '05, 2005, pp. 488495.

[25] T. B. Sebastian, P. N. Klein, and B. B. Kimia, "Alignment-based recognition of shape outlines," in IWVF-4: Proceedings of the 4th International Workshop on Visual Form. London, UK: SpringerVerlag, 2001, pp. 606-618.

[26] R. C. Veltkamp and M. Hagedoorn, State of the art in shape matching. London, UK: Springer-Verlag, 2001, pp. 87-119.

[27] D. Douglas and T. Peucker, "Algorithms for the reduction of the number of points required to represent a digitized line or its caricature," Canadian Cartographer, vol. 10,, no. 2, pp. 112-122, 1973.

[28] A. Finkelstein and D. H. Salesin, "Multiresolution curves," in Proc. SIGGRAPH '94, 1994, pp. 261-268.

[29] P. Barla, J. Thollot, and F. Sillion, "Geometric clustering for line drawing simplification," in Proc. Eurographics Symposium on Rendering, 2005

[30] P. V. Sander, X. Gu, S. J. Gortler, H. Hoppe, and J. Snyder, "Silhouette clipping," in Proc. SIGGRAPH '00, New York, NY, USA, 2000, pp. 327-334.

[31] B. Aronov, R. Seidel, and D. Souvaine, "On compatible triangulations of simple polygons," Computational Geometry: Theory and Applications, vol. 3, pp. 27-35, 1993.

[32] H. Gupta and R. Wenger, "Constructing piecewise linear homeomorphisms of simple polygons," J. Algorithms, vol. 22, no. 1, pp. 142-157, 1997.

[33] S. Suri, "On some link distance problems in a simple polygon," IEEE Trans. Robotics and Automation, vol. 6, no. 1, pp. 108-113, Feb 1990.

[34] — "A linear time algorithm with minimum link paths inside a simple polygon," CVGIP, vol. 35, no. 1, pp. 99-110, 1986.

[35] L. Guibas, J. Hershberger, D. Leven, M. Sharir, and R. Tarjan, "Linear time algorithms for visibility and shortest path problems inside simple polygons," in SCG '86: Proc. Symposium on Comp. Geom., 1986, pp. 1-13.

[36] L. Guibas, E. McCreight, M. Plass, and J. Roberts, "A new representation for linear lists," in Proc. 9th ACM Symp. on Theory of Computing, 1977, pp. 49-60.

[37] B. Chazelle, "Triangulating a simple polygon in linear time," Discrete Comp. Geom., vol. 6, no. 5, pp. 485-524, 1991.

[38] N. M. Amato, M. T. Goodrich, and E. A. Ramos, "A randomized algorithm for triangulating a simple polygon in linear time," Discrete \& Comp. Geometry, vol. 26, no. 2, pp. 245-265, 2001.

[39] B. Joe and R. B. Simpson, "Corrections to Lee's visibility polygon algorithm," BIT, vol. 27, no. 4, pp. 458-473, 1987.

[40] C. A. Hipke, "Computing visibility polygons with LEDA," 1996. [Online]. Available: http://ad.informatik.uni-freiburg.de/ mitarbeiter/hipke/_hipke.work.vis_polygon.ps.gz

[41] Bonn, University of, "Applets for visibility in simple polygons," 2008. [Online]. Available: http://web.informatik.uni-bonn.de/I/ GeomLab/VisPolygon/

[42] H. Hoppe, "Progressive meshes," Proc. SIGGRAPH '96, pp. 98$108,1996$.

[43] P. V. Sander, J. Snyder, S. J. Gortler, and H. Hoppe, "Texture mapping progressive meshes," in Proc. SIGGRAPH' 01, 2001, pp. 409-416.

[44] W. Baxter, P. Barla, and K. Anjyo, "Notes on compatible mapping of 2D shapes," OLM Digital, Inc., Tech. Rep. OLMTRE-2008-002, 2008. [Online]. Available: http://www.olm.co.jp/en/rd/2008/ 06/compatible embedding notes.html

[45] W. Baxter, P. Barla, and K. ichi Anjyo, "Rigid shape interpolation using normal equations," in Proc. Symp. Non-Photorealistic Animation and Rendering, 2008, pp. 59-64.

[46] V. Surazhsky, personal communication, June 2008.

[47] W. Baxter and K. Anjyo, "Latent doodle space," Computer Graphics Forum, vol. 25, no. 3, pp. 477-485, 2006. 

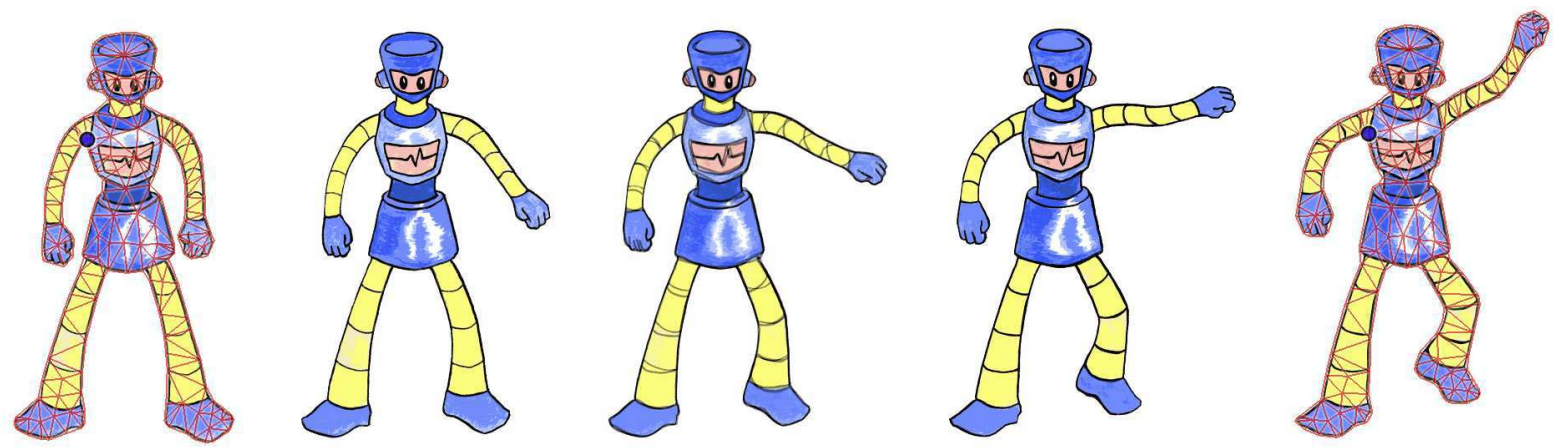

Fig. 11. Simple morphing result: Morphing result using compatible meshes generated by our techniques, with linear texture blending. First and last frames show triangulations. This result required just a single boundary correspondence (blue dot). The original inputs were hand-drawn by an artist.

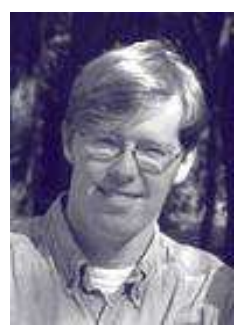

William Baxter received the $\mathrm{PhD}$ in computer science from the University of North Carolina at Chapel Hill in 2004. He has been employed as a Senior Researcher in the R\&D group at OLM Digital, Inc. in Tokyo, Japan since January 2005. His main research interests are NPR, $\mathrm{HCl}$, and physically based models, with a particular interest in how these can be used to enhance and support creative activities.

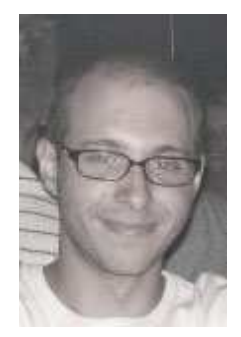

Pascal Barla received the $\mathrm{PhD}$ in computer science from INRIA Grenoble University in 2006 , and has recently started as a researcher at INRIA Bordeaux Sud-Ouest, in the IPARLA team. His main research interest is expressive rendering, with a strong interest in human vision.

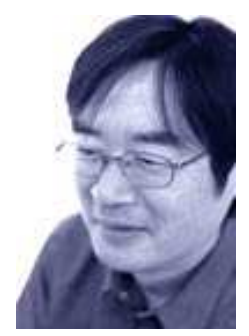

Ken-ichi Anjyo received the $\mathrm{PhD}$ in information engineering from Nagoya University in 1994, and has been working for OLM Digital, Inc., since 2000. At OLM Digital he is the lead of the Research and Development division, responsible for making visual effects and in-house software tools. His research focus is the construction of mathematical and computationally tractable models for computer graphics. 

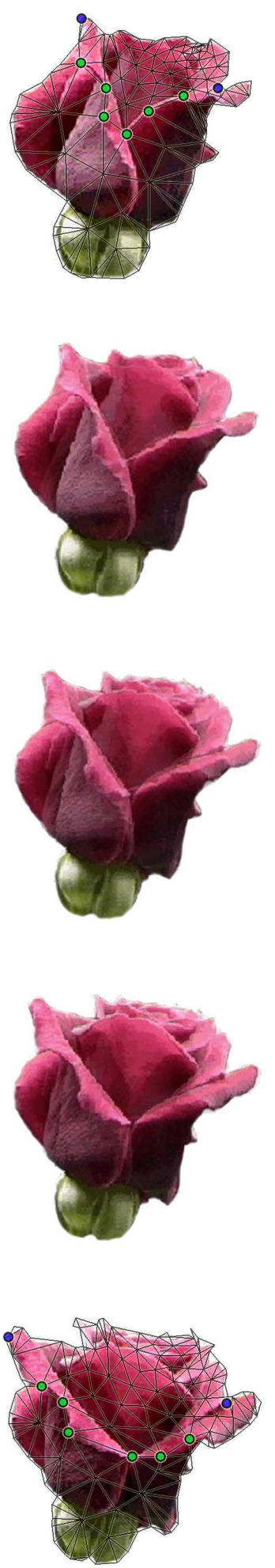

- 2 BCs / O 6 ICs
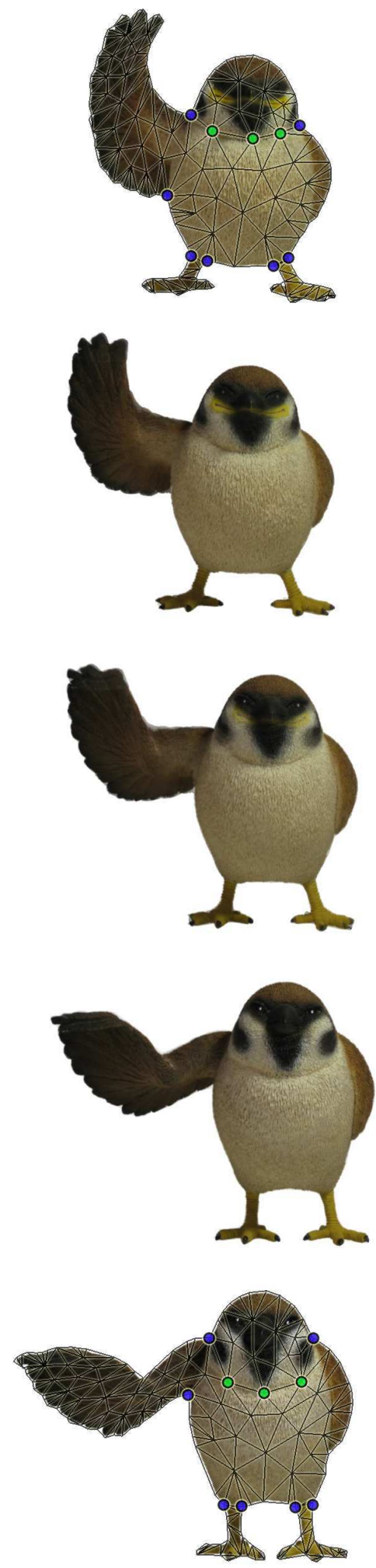

- 7 BCs / O 3 ICs
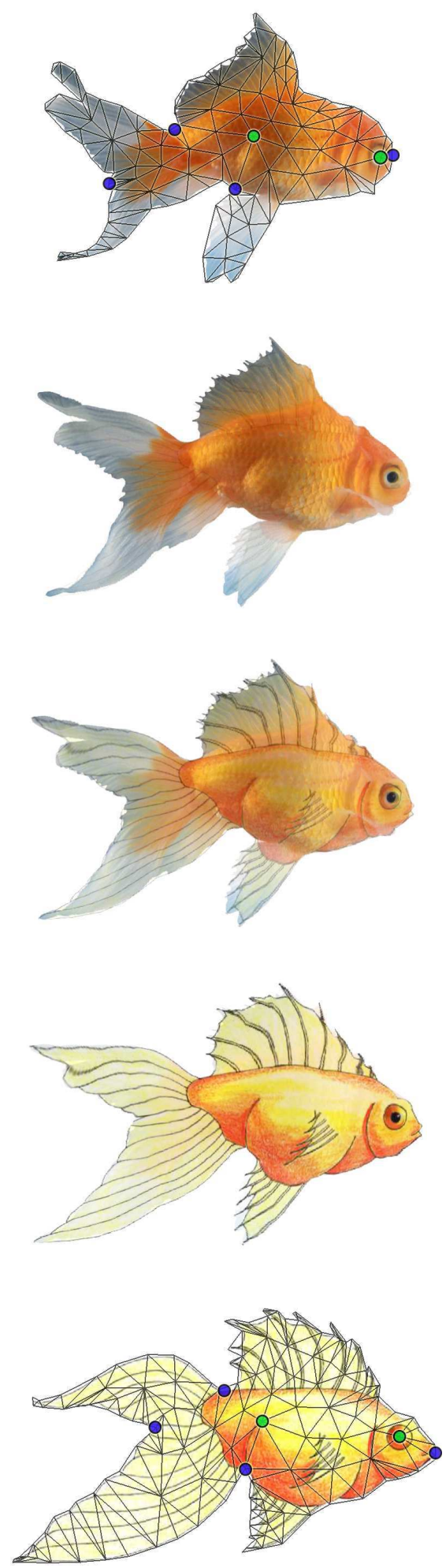

- 4 BCs / O 2 ICs

Fig. 12. Additional morphing results: Morphing results on more complex inputs. Boundary (BC) and interior (IC) correspondences are indicated. 